\title{
Oncogene MAX
}

National Cancer Institute

\section{Source}

National Cancer Institute. Oncogene MAX. NCI Thesaurus. Code C18604.

Oncogene MAX is a member of the MYC gene family. The MAX gene encodes a bHLH/ZIP protein that forms a heterodimer with MYC and binds with high affinity to a DNA site in the MYC gene and DDX18, a DEAD box family member. MAX mRNA steady state level is not modulated with respect to cell proliferation or differentiation, unlike mRNAs encoded by most immediate early genes. MAX also interacts with MIX2. The human gene is located at $14 \mathrm{q} 23$. 